\title{
On global smooth solutions to the 3D Vlasov-Nordström system
}

\section{Sur les solutions régulières du système de Vlasov-Nordström tridimensionnel}

\author{
Christophe Pallard \\ D.M.A., École Normale Supérieure, 45, rue d'Ulm, 75230 Paris cedex 05, France \\ Received 8 June 2004; received in revised form 21 July 2004; accepted 2 February 2005 \\ Available online 20 April 2005
}

\begin{abstract}
The Vlasov-Nordström system is a relativistic model describing the motion of a self-gravitating collisionless gas. A conditional existence result for global smooth solutions was obtained in [Comm. Partial Differential Equations 28 (2003) 1863-1885]. We give a new proof for this result.

(C) 2006 L'Association Publications de l'Institut Henri Poincaré. Published by Elsevier B.V. All rights reserved
\end{abstract}

\section{Résumé}

Le système de Vlasov-Nordström est un modèle relativiste décrivant l'évolution d'un ensemble de particules massives soumises au champ gravitationnel qu'elles génèrent collectivement. Un théorème d'existence conditionnelle a été démontré dans [Comm. Partial Differential Equations 28 (2003) 1863-1885]. Nous donnons ici une nouvelle preuve de ce résultat.

(C) 2006 L'Association Publications de l'Institut Henri Poincaré. Published by Elsevier B.V. All rights reserved

MSC: $85 \mathrm{~A} 05 ; 82 \mathrm{C} 22$

\section{Introduction}

\subsection{The Vlasov-Nordström system}

This is a relativistic kinetic model describing the behaviour of a collisionless set of particles interacting through gravitational forces. It may be thought of as a relativistic generalization of the Vlasov-Poisson system, the latter being obtained as its Newtonian limit [5]. Using the framework of Nordström's theory [11], whereby gravitational

\footnotetext{
E-mail address: pallard@dma.ens.fr.
} 
effects are mediated by a scalar field, the Vlasov-Nordström system is a much simpler model than the VlasovEinstein system. Nevertheless, as it couples Vlasov equation with a hyperbolic equation, it remains less well understood than the standard Vlasov-Poisson system. For more background and references, we refer to [4], where a thorough derivation of the Vlasov-Nordström system can be found. See also [1,6-8,14]. We shall consider the following formulation. The unknowns are functions $f \equiv f(t, x, \xi) \geqslant 0$ and $\phi \equiv \phi(t, x)$ with $(t, x, \xi) \in \mathbf{R}_{+} \times \mathbf{R}^{3} \times \mathbf{R}^{3}$, satisfying Vlasov equation

$$
T f=\nabla_{\xi} \cdot\left[\left((T \phi) \xi+\frac{\nabla_{x} \phi}{\sqrt{1+|\xi|^{2}}}\right) f\right]+f T \phi,
$$

$T$ being the streaming operator $T=\partial_{t}+v(\xi) \cdot \nabla_{x}$ and $v$ the relativistic velocity of a particle of momentum $\xi$ :

$$
v(\xi)=\frac{\xi}{\sqrt{1+|\xi|^{2}}} .
$$

The scalar field $\phi$ is supposed to solve the wave equation

$$
\square_{t, x} \phi=-\mu,
$$

with

$$
\mu=\int \frac{f \mathrm{~d} \xi}{\sqrt{1+|\xi|^{2}}} .
$$

The Cauchy problem for the Vlasov-Nordström system (VN) consists in Eqs. (1.1), (1.2) and (1.3) together with initial data

$$
f_{\mid t=0}=f_{I}, \quad \phi_{\mid t=0}=\phi_{I}, \quad \partial_{t} \phi_{\mid t=0}=\phi_{I}^{\prime} .
$$

In these equations, all physical constants have been set equal to unity. The interpretation of a solution $(f, \phi)$ is the following: the space-time is a Lorentzian manifold with a conformally flat metric given in coordinates $(t, x)$ by

$$
g_{\mu \nu}=\mathrm{e}^{2 \phi} \operatorname{diag}(-1,1,1,1)
$$

and the particle density on the mass shell in this metric is $\mathrm{e}^{-4 \phi} f\left(t, x, \mathrm{e}^{\phi} \xi\right)$.

This system should be compared to another kinetic model arising in plasma physics, the relativistic VlasovMaxwell system (RVM), which describes the behaviour of a collisionless set of charged particles interacting through a self-generated electromagnetic field. In particular, it is known since Glassey and Strauss [10]-and reproved in $[3,13]$ - that smooth solutions to (RVM) do not develop singularities as long as the momentum of particles remains bounded. The corresponding result for $(\mathrm{VN})$ was shown in [6,7] by similar means. Defining the size of the momentum support as

$$
R(t)=\sup \left\{|\xi|: \exists x \in \mathbf{R}^{3} f(t, x, \xi) \neq 0\right\},
$$

we have the following theorem, established in $[6,7]$.

Theorem 1.1. Let $\tau>0$. Let $f \in \mathcal{C}^{1}\left([0, \tau) \times \mathbf{R}^{3} \times \mathbf{R}^{3}\right)$ and $\phi \in \mathcal{C}^{2}\left([0, \tau) \times \mathbf{R}^{3}\right)$ be a solution of $(\mathrm{VN})$ with initial data $f_{I} \in \mathcal{C}_{c}^{1}\left(\mathbf{R}^{3} \times \mathbf{R}^{3}\right), \phi_{I} \in \mathcal{C}_{c}^{3}\left(\mathbf{R}^{3}\right)$ and $\phi_{I}^{\prime} \in \mathcal{C}_{c}^{2}\left(\mathbf{R}^{3}\right)$. Then for any $t \in[0, \tau]$ we have

$$
\sup _{s \in[0, t)} R(s)<+\infty \quad \Longrightarrow \quad\|f\|_{W^{1, \infty}\left([0, t) \times \mathbf{R}^{6}\right)}+\|\phi\|_{W^{2, \infty}\left([0, t) \times \mathbf{R}^{3}\right)}<+\infty .
$$

A corollary of this result is that if a smooth solution blows up in finite time then $R$ becomes infinite. For if it were not the case, the estimates (1.6) would allow to extend the solution as described in [6], p. 1881. The proof of theorem 1.1 in [6] relies essentially on the same procedures than those found in [10]. In this paper, we give a new proof by handling the fields and their derivatives using a method similar to [3], where an alternative derivation of the Glassey-Strauss' theorem is performed. 


\subsection{Kinetic formulation}

The starting point in [3] is an adequate 'kinetic formulation' of the system, which was introduced in [2]. Let us show why this approach is relevant in the context of the Vlasov-Nordström system. Introduce a scalar potential $u \equiv u(t, x, \xi)$ solving the wave equation

$$
\square_{t, x} u=f, \quad u_{\mid t=0}=0, \quad \partial_{t} u_{\mid t=0}=0 .
$$

Let $\phi^{0}$ be the solution to

$$
\square_{t, x} \phi^{0}=0, \quad \phi_{\mid t=0}^{0}=\phi_{I}, \quad \partial_{t} \phi_{\mid t=0}^{0}=\phi_{I}^{\prime} .
$$

And define

$$
\begin{aligned}
\phi_{u} & =\phi^{0}-\int \frac{u \mathrm{~d} \xi}{\sqrt{1+|\xi|^{2}}}, \\
K_{u} & =\left(T \phi_{u}\right) \xi+\frac{\nabla_{x} \phi_{u}}{\sqrt{1+|\xi|^{2}}} .
\end{aligned}
$$

Then the Vlasov-Nordström system $(\mathrm{VN})$ is equivalent to

$$
\begin{aligned}
& \square_{t, x} u=f, \\
& T f=\nabla_{\xi} \cdot\left(f K_{u}\right)+f T \phi_{u},
\end{aligned}
$$

with initial data

$$
f_{\mid t=0}=f_{0}, \quad u_{\mid t=0}=0, \quad \partial_{t} u_{\mid t=0}=0 .
$$

This representation of the scalar field $\phi_{u}$ as a $\xi$ average of $u$ allows a treatment similar to [3]. That is, we derive suitable expressions of the derivatives of $\phi_{u}$ by working on the fundamental solution of the wave operator. The benefits of this approach are a unified treatment for all derivatives as well as a natural explanation for a key point in both the present paper and [6], namely the vanishing average of some particular coefficients. We also mention that this method extends to the two-dimensional case studied in [14], see the remarks in [3] on this question. In the next section we recall the so-called division lemma, on which we shall rely heavily. Section 3 is devoted to establishing estimates on $f, \phi_{u}$ and their derivatives leading to the proof of Theorem 1.1. We use standard notations. In inequalities, constants that depend on some parameters $\lambda_{1}, \ldots, \lambda_{k}$ are denoted by $C\left(\lambda_{1}, \ldots, \lambda_{k}\right)$ and may change from line to line.

\section{A division lemma}

Let $Y \in \mathcal{D}^{\prime}\left(\mathbf{R}^{4}\right)$ be the forward fundamental solution of the wave operator:

$$
Y(t, x)=\frac{\mathbf{1}_{t>0}}{4 \pi t} \delta(|x|-t) .
$$

Notice that the distribution $Y$ is homogeneous of degree -2 in $\mathbf{R}^{4}$. Let $\mathcal{M}_{m}$ be the space of $C^{\infty}$ homogeneous functions of degree $m$ on $\mathbf{R}^{4} \backslash 0$. Below, we use the notation

$$
x_{0}:=t, \quad \text { and } \quad \partial_{j}:=\partial_{x_{j}}, \quad j=0, \ldots, 3 .
$$

The following lemma can be found almost verbatim in [3].

Lemma 2.1 (Division lemma). For each $\xi \in \mathbf{R}^{3}$, 
- there exists functions $a_{i}^{k} \equiv a_{i}^{k}(t, x)$ where $i=0, \ldots, 3$ and $k=0,1$, such that $a_{i}^{k} \in \mathcal{M}_{-k}$ and

$$
\partial_{i} Y=T\left(a_{i}^{0} Y\right)+a_{i}^{1} Y, \quad i=0, \ldots, 3 ;
$$

- there exists functions $b_{i j}^{k} \equiv b_{i j}^{k}(t, x)$ with $i, j=0, \ldots, 3, k=0,1,2$, such that $b_{i j}^{k} \in \mathcal{M}_{-k}$ and

$$
\partial_{i j}^{2} Y=T^{2}\left(b_{i j}^{0} Y\right)+T\left(b_{i j}^{1} Y\right)+b_{i j}^{2} Y, \quad i, j=0, \ldots, 3 ;
$$

- moreover, the functions $b_{i j}^{2}$ satisfy the conditions

$$
\int_{\mathbf{S}^{2}} b_{i j}^{2}(1, y) \mathrm{d} \sigma(y)=0, \quad i, j=0, \ldots, 3,
$$

where $\mathrm{d} \sigma(y)$ is the rotation invariant surface element on the unit sphere $\mathbf{S}^{2}$ of $\mathbf{R}^{3}$. In both formulas (2.3) and (2.4), $a_{i}^{0} Y, a_{i}^{1} Y, b_{i j}^{0} Y$ and $b_{i j}^{1} Y$ designate, for each $i, j=0, \ldots, 3$, the unique extensions as homogeneous distributions on $\mathbf{R}^{4}$ of those same expressions-which are a priori only defined on $\mathbf{R}^{4} \backslash 0$. Likewise, $b_{i j}^{2} Y$ designates, for $i, j=0, \ldots, 3$ the unique extension as a homogeneous distribution of degree -4 on $\mathbf{R}^{4}$ of that same expressions for which the relation (2.4) holds in the sense of distributions on $\mathbf{R}^{4}$.

\section{Remarks.}

1. The proof of Lemma 2 is in [3]. It is based on the commutation properties of the wave operator with the Lorentz boosts.

2. We refer the reader to the reference for the expressions of coefficients $a_{i}^{k}(t, x, \xi)$ and $b_{i j}^{k}(t, x, \xi)$. In the sequel, all we shall need are the following two properties: $a_{i}^{k}, b_{i j}^{k} \in \mathcal{C}^{\infty}\left(\mathbf{R}^{4} \backslash 0 \times \mathbf{R}^{3}\right)$ and for any $\xi \in \mathbf{R}^{3}$ and $\alpha \in \mathbf{N}^{3}$ we have $\partial_{\xi}^{\alpha} a_{i}^{k}(\cdot, \cdot, \xi) \in \mathcal{M}_{-k}$ and $\partial_{\xi}^{\alpha} b_{i j}^{k}(\cdot, \cdot, \xi) \in \mathcal{M}_{-k}$.

3. We recall here some facts about homogeneous distributions. Any homogeneous distribution of degree $k>-3$ on $\mathbf{R}^{4} \backslash 0$ has a unique extension on $\mathbf{R}^{4}$ that is also homogeneous of degree $k$. A homogeneous distribution of degree -4 on $\mathbf{R}^{4} \backslash 0$ may not be extendable on $\mathbf{R}^{4}$. If such a homogeneous extension exists, then it is not unique: two extensions may differ by a multiple of $\delta_{x=0}$. For more details, see the appendix of [3] and references therein $[9,12]$.

\section{Proof of Theorem 1.1}

\subsection{Estimates on $f$}

We begin by showing that the needed estimates on $f$ and its first derivatives will follow from estimates on $\phi_{u}$. This is done by working on the transport equation satisfied by $f$. Following [6], we thus rewrite (1.12) as

$$
\begin{aligned}
T\left(\mathrm{e}^{-4 \phi_{u}} f\right) & =-4 \mathrm{e}^{-4 \phi_{u}} f T \phi_{u}+\mathrm{e}^{-4 \phi_{u}} T f \\
& =-4 \mathrm{e}^{-4 \phi_{u}} f T \phi_{u}+\mathrm{e}^{-4 \phi_{u}}\left(\nabla_{\xi} \cdot\left(f K_{u}\right)+f T \phi_{u}\right) \\
& =-3 \mathrm{e}^{-4 \phi_{u}} f T \phi_{u}+K_{u} \cdot \nabla_{\xi}\left(\mathrm{e}^{-4 \phi_{u}} f\right)+\mathrm{e}^{-4 \phi_{u}} f \nabla_{\xi} \cdot K_{u} .
\end{aligned}
$$

The expression of $K_{u}$ gives

$$
\begin{aligned}
\nabla_{\xi} \cdot K_{u} & =\nabla_{\xi} \cdot\left(T \phi_{u} \xi+\frac{\nabla_{x} \phi_{u}}{\sqrt{1+|\xi|^{2}}}\right) \\
& =\left(\xi \cdot \nabla_{\xi}\right)\left(v \cdot \nabla_{x} \phi_{u}\right)+3 T \phi_{u}+\left(\nabla_{x} \phi_{u}\right) \cdot \nabla_{\xi}\left(\frac{1}{\sqrt{1+|\xi|^{2}}}\right) .
\end{aligned}
$$


A short computation shows that

$$
\left(\xi \cdot \nabla_{\xi}\right)\left(v \cdot \nabla_{x} \phi_{u}\right)=\frac{v \cdot \nabla_{x} \phi_{u}}{1+|\xi|^{2}}
$$

and

$$
\left(\nabla_{x} \phi_{u}\right) \cdot \nabla_{\xi}\left(\frac{1}{\sqrt{1+|\xi|^{2}}}\right)=-\frac{v \cdot \nabla_{x} \phi_{u}}{1+|\xi|^{2}} .
$$

So that we find

$$
T\left(\mathrm{e}^{-4 \phi_{u}} f\right)-\left(T \phi_{u} \xi+\frac{\nabla_{x} \phi_{u}}{\sqrt{1+|\xi|^{2}}}\right) \cdot \nabla_{\xi}\left(\mathrm{e}^{-4 \phi_{u}} f\right)=0 .
$$

The characteristic curves of this equation remain the same as those derived from (1.12). These are curves $t \mapsto(X(t), \Xi(t))$ satisfying

$$
\begin{aligned}
& X^{\prime}(t)=v(\Xi(t)), \\
& \Xi^{\prime}(t)=-\left(T \phi_{u}\right)(t, X(t), \Xi(t)) \Xi(t)-\frac{\left(\nabla_{x} \phi_{u}\right)(t, X(t), \Xi(t))}{\sqrt{1+|\Xi(t)|^{2}}},
\end{aligned}
$$

with initial data $X(0)=x_{0}$ and $\Xi(0)=\xi_{0}$. We infer from (3.1) that $\mathrm{e}^{-4 \phi_{u}} f$ is constant along these curves and we get equality (2.7) of [6]:

$$
f(t, X(t), \Xi(t))=f_{I}\left(x_{0}, \xi_{0}\right) \exp \left(4 \phi_{u}(t, X(t))-4 \phi_{I}\left(x_{0}\right)\right) .
$$

As was observed in [7], $u$ solves the wave equation (1.7) with a right-hand side $f \geqslant 0$ and vanishing initial data, so that $u \geqslant 0$. From (1.9), it comes $\phi_{u} \leqslant \phi^{0}$ and we recover proposition 1 of [7]:

$$
\|f(t, \cdot, \cdot)\|_{L^{\infty}} \leqslant C\left(f_{I}, \phi_{I}, \phi_{I}^{\prime}, \tau\right) .
$$

A look at (3.2) shows that since $f_{I}$ is compactly supported, the momentum support of $f(t, \cdot, \cdot)$ remains bounded for any $t<\tau$. From now on, we assume

$$
\sup _{t \in[0, \tau)} R(t)=r^{*}<+\infty .
$$

Differentiating equality (1.12) in $x$ or $\xi$, we find

$$
T(D f)-\nabla_{\xi} \cdot\left((D f) K_{u}\right)=[T, D] f+\nabla_{\xi} \cdot\left(f D K_{u}\right)+D\left(f T \phi_{u}\right),
$$

where $D$ denotes $\partial_{x_{i}}$ or $\partial_{\xi_{i}}$. Therefore with (3.3),

$$
\begin{aligned}
& \|f(t, \cdot, \cdot)\|_{W^{1, \infty}} \\
& \quad \leqslant C\left(f_{I}, \phi_{I}, \phi_{I}^{\prime}, \tau, r^{*}\right)\left(1+\int_{0}^{t}\|f(s, \cdot, \cdot)\|_{W^{1, \infty}}\left(1+\left\|\phi_{u}(s, \cdot)\right\|_{W^{2, \infty}}+\left\|\partial_{t} \phi_{u}(s, \cdot)\right\|_{W^{1, \infty}}\right) \mathrm{d} s\right) .
\end{aligned}
$$

The next three subsections are devoted to estimating $\phi_{u}$, its first and second derivatives. Note that we aim at using inequality (3.5) with Gronwall's lemma. This requires bounds that do not grow too fast with respect to the quantity $\|f(t, \cdot, \cdot)\|_{W^{1, \infty}}$. 


\subsection{Bound on $\phi_{u}$}

The easiest one. We have to estimate

$$
\phi_{u}=\phi^{0}-\int \frac{u \mathrm{~d} \xi}{\sqrt{1+|\xi|^{2}}} .
$$

We recall the following elementary inequalities for the wave equation

$$
\left\|\phi^{0}\right\|_{W^{k, \infty}\left([0, t] \times \mathbf{R}^{3}\right)} \leqslant(1+t)\left\|\phi_{I}\right\|_{W^{k+1, \infty}}+t\left\|\phi_{I}^{\prime}\right\|_{W^{k, \infty}} .
$$

Thus the first term in (3.6) can be estimated by

$$
\left\|\phi^{0}(t, \cdot)\right\|_{L^{\infty}} \leqslant(1+t)\left\|\phi_{I}\right\|_{W^{1, \infty}}+t\left\|\phi_{I}^{\prime}\right\|_{L^{\infty}} .
$$

Let $\chi \in \mathcal{C}_{c}^{\infty}\left(\mathbf{R}^{3}\right)$ be a cut-off function such that $\chi(\xi)=1$ when $|\xi| \leqslant r^{*}$ and vanishing when $|\xi|>2 r^{*}$. Define

$$
m(\xi)=\frac{1}{\sqrt{1+|\xi|^{2}}} \chi(\xi) \text {. }
$$

From relation (1.7), we know that the momentum support of $u$ and $f$ are equal. Therefore the second term in (3.6) satisfy

$$
\int \frac{u(t, x, \xi) \mathrm{d} \xi}{\sqrt{1+|\xi|^{2}}}=\int m(\xi) u(t, x, \xi) \mathrm{d} \xi
$$

The function $u$ solves the wave equation (1.7), so that ${ }^{1}$

$$
u=Y \star\left(f \mathbf{1}_{t>0}\right) .
$$

And since $Y(t, \cdot)$ is a positive measure of total mass $t$, it comes

$$
\left\|\int m(\xi) u(t, \cdot, \xi) \mathrm{d} \xi\right\|_{L^{\infty}} \leqslant \frac{4}{3} \pi r^{* 3} \int_{0}^{t}(t-s)\|f(s, \cdot, \cdot)\|_{L^{\infty}} \mathrm{d} s .
$$

With (3.3), we find

$$
\left\|\phi_{u}(t, \cdot)\right\|_{L^{\infty}} \leqslant C\left(f_{I}, \phi_{I}, \phi_{I}^{\prime}, \tau, r^{*}\right) .
$$

\subsection{Bounds on first derivatives of $\phi_{u}$}

We intend here to estimate

$$
I(t)=\sup _{i=0, \ldots, 3}\left\|\partial_{i} \phi_{u}(t, \cdot)\right\|_{L^{\infty}} .
$$

Derivating (3.6), we find

$$
\partial_{i} \phi_{u}(t, x)=\partial_{i} \phi^{0}(t, x)-\partial_{i} \int m(\xi) u(t, x, \xi) \mathrm{d} \xi,
$$

for $i=0, \ldots, 3$. The first term is estimated with (3.7). It comes

$$
\left\|\partial_{i} \phi^{0}(t, \cdot)\right\|_{L^{\infty}} \leqslant C\left(\phi_{I}, \phi_{I}^{\prime}, t\right)
$$

\footnotetext{
$\overline{1}$ In the sequel, $\star$ denotes convolution in the space and time variables, while $\star_{x}$ denotes convolution in the space variable only.
} 
Consider now the second term. In view of the remark following (3.5), straightforward estimates on $\partial_{i} u=Y \star$ $\partial_{i}\left(f \mathbf{1}_{t>0}\right)$ would not lead to interesting bounds. Instead, we use (3.8) with Lemma 2.1 to get

$$
\partial_{i} u=\left(a_{i}^{1} Y\right) \star\left(f \mathbf{1}_{t>0}\right)+\left(a_{i}^{0} Y\right) \star T\left(f \mathbf{1}_{t>0}\right) .
$$

Besides, we infer from equation (1.12)

$$
T\left(f \mathbf{1}_{t>0}\right)=(T f) \mathbf{1}_{t>0}+f_{I} \delta_{t=0}=\nabla_{\xi} \cdot\left(f K_{u}\right) \mathbf{1}_{t>0}+f\left(T \phi_{u}\right) \mathbf{1}_{t>0}+f_{I} \delta_{t=0} .
$$

It only remains to get rid of derivatives in the $\xi$ variable by integrating by parts, leading eventually to the expression:

$$
\begin{aligned}
\partial_{i} \int m(\xi) u(t, x, \xi) \mathrm{d} \xi= & \int m(\xi)\left(\left(a_{i}^{1} Y\right) \star\left(f \mathbf{1}_{t>0}\right)\right)(t, x, \xi) \mathrm{d} \xi \\
& +\int m(\xi)\left(\left(a_{i}^{0} Y(t, \cdot)\right) \star_{x} f_{I}\right)(x, \xi) \mathrm{d} \xi \\
& +\int\left(\left(-\nabla_{\xi}\left(m a_{i}^{0}\right) Y\right) \star\left(f \mathbf{1}_{t>0} K_{u}\right)\right)(t, x, \xi) \mathrm{d} \xi \\
& +\int\left(\left(m a_{i}^{0} Y\right) \star\left(f \mathbf{1}_{t>0} T \phi_{u}\right)\right)(t, x, \xi) \mathrm{d} \xi .
\end{aligned}
$$

The interest of Lemma 2.1 is now obvious: we don't need to differentiate $f$ in the previous decomposition. Repeatedly using the fact that $Y(t, \cdot)$ is a positive measure of total mass $t$, we get

$$
\begin{aligned}
I(t) \leqslant & C\left(\phi_{I}, \phi_{I}^{\prime}, t\right)+\frac{4}{3} \pi r^{* 3}\left(\left\|m t a_{i}^{1}\right\|_{L^{\infty}} \int_{0}^{t}\|f(s, \cdot, \cdot)\|_{L^{\infty}} \mathrm{d} s+\left\|m a_{i}^{0}\right\|_{L^{\infty}} t\left\|f_{I}\right\|_{L^{\infty}}\right. \\
& \left.+\left\|m a_{i}^{0}\right\|_{L_{t, x}^{\infty}\left(W_{\xi}^{1, \infty}\right)} \int_{0}^{t}(t-s)\left\|f K_{u}(s, \cdot, \cdot)\right\|_{L^{\infty}} \mathrm{d} s+\left\|m a_{i}^{0}\right\|_{L^{\infty}} \int_{0}^{t}(t-s)\left\|f T \phi_{u}(s, \cdot, \cdot)\right\|_{L^{\infty}} \mathrm{d} s\right) .
\end{aligned}
$$

It follows from expression (1.10) that

$$
\left\|K_{u}(s, \cdot, \cdot)\right\|_{L^{\infty}\left(\mathbf{R}^{3} \times B\left(0, r^{*}\right)\right)} \leqslant C\left(r^{*}\right) I(s) .
$$

With inequality (3.3) and expression (1.9), we find

$$
I(t) \leqslant C\left(f_{I}, \phi_{I}, \phi_{I}^{\prime}, \tau, r^{*}\right)\left(1+\int_{0}^{t} I(s) \mathrm{d} s\right) .
$$

Applying Gronwall's lemma to inequality (3.12), it comes

$$
\sup _{t \in[0, \tau)} I(t) \leqslant C\left(f_{I}, \phi_{I}, \phi_{I}^{\prime}, \tau, r^{*}\right) .
$$

\subsection{Bounds on second derivatives of $\phi_{u}$}

We define

$$
J(t)=\sup _{i, j=0, \ldots, 3}\left\|\partial_{i j} \phi_{u}(t, \cdot)\right\|_{L^{\infty}} .
$$

Differentiating (3.6) twice,

$$
\partial_{i j} \phi_{u}(t, x)=\partial_{i j} \phi^{0}(t, x)+\partial_{i j} \int m(\xi) u(t, x, \xi) \mathrm{d} \xi,
$$


for any $i, j=0, \ldots, 3$. From (3.7), it comes

$$
\left\|\partial_{i j} \phi^{0}(t, \cdot)\right\|_{L^{\infty}} \leqslant C\left(\phi_{I}, \phi_{I}^{\prime}, t\right) .
$$

Using (3.8) and Lemma 2.1,

$$
\begin{aligned}
\partial_{i j} \int m(\xi) u(t, x, \xi) \mathrm{d} \xi= & \int m(\xi)\left(\left(b_{i j}^{2} Y\right) \star\left(f \mathbf{1}_{t>0}\right)\right)(t, x, \xi) \mathrm{d} \xi+\int m(\xi)\left(\left(b_{i j}^{1} Y\right) \star T\left(f \mathbf{1}_{t>0}\right)\right)(t, x, \xi) \mathrm{d} \xi \\
& +\int m(\xi)\left(\left(b_{i j}^{0} Y\right) \star T^{2}\left(f \mathbf{1}_{t>0}\right)\right)(t, x, \xi) \mathrm{d} \xi=S_{0}+S_{1}+S_{2} .
\end{aligned}
$$

Estimates for $S_{0}$. The key point here is the fact that the average of the coefficients $b_{i j}^{2}$ vanishes, which allows us to obtain sharp estimates for $S_{0}$. As will be seen below, the contribution of this term to $J(t)$ is crucial. First, let us determine a homogeneous extension of $b_{i j}^{2} Y$ on $\mathbf{R}^{4}$. Let $\phi \in \mathcal{C}_{c}^{\infty}\left(\mathbf{R}^{4} \backslash 0\right)$ be a test function and consider

$$
\left\langle b_{i j}^{2} Y, \phi\right\rangle=\int_{0|y|=1}^{\infty} \int_{i j}^{2}(1, y, \xi) \phi(t, t y) \frac{\mathrm{d} S_{y}}{4 \pi t} \mathrm{~d} t,
$$

where we used the homogeneity of $b_{i j}^{2}(\cdot, \cdot, \xi) \in \mathcal{M}_{-2}$ for any $\xi$. Since $b_{i j}^{2}$ satisfy (2.5), the following equality holds for any $\theta \geqslant 0$ :

$$
\left\langle b_{i j}^{2} Y, \phi\right\rangle=\int_{0}^{\theta} \int_{|y|=1} b_{i j}^{2}(1, y, \xi)(\phi(t, t y)-\phi(t, 0)) \frac{\mathrm{d} S_{y}}{4 \pi t} \mathrm{~d} t+\int_{\theta|y|=1}^{\infty} \int_{i j} b^{2}(1, y, \xi) \phi(t, t y) \frac{\mathrm{d} S_{y}}{4 \pi t} \mathrm{~d} t .
$$

But the right-hand side of (3.15) still makes sense for test functions on $\mathbf{R}^{4}$. Denote by p.v. $\left(b_{i j}^{2} Y\right)$ the distribution defined by this expression. ${ }^{2}$ This is a homogeneous distribution of degree -4 on $\mathbf{R}^{4}$ that extends $b_{i j}^{2} Y$. It follows from the third remark in Section 2 the relation

$$
b_{i j}^{2} Y-\text { p.v. }\left(b_{i j}^{2} Y\right)=c(\xi) \delta_{(t, x)=(0,0)},
$$

where $c_{i j} \in \mathcal{C}^{\infty}\left(\mathbf{R}^{3}\right)$; indeed, the left-hand side of this equality is smooth as a function of $\xi-$ see the second remark below the lemma. Thus, for $\theta_{t}$ to be chosen later,

$$
\begin{aligned}
S_{0}- & \int m(\xi) c_{i j}(\xi) f(t, x, \xi) \mathrm{d} \xi=\int m(\xi)\left(\text { p.v. }\left(b_{i j}^{2} Y\right) \star\left(f \mathbf{1}_{t>0}\right)\right)(t, x, \xi) \mathrm{d} \xi \\
= & \int m(\xi) \int_{0}^{\theta_{t}} \int_{|y|=1} b_{i j}^{2}(1, y, \xi)(f(t-s, x-s y, \xi)-f(t-s, x, \xi)) \frac{\mathrm{d} S_{y}}{4 \pi s} \mathrm{~d} s \mathrm{~d} \xi \\
& +\int m(\xi) \int_{\theta_{t}}^{t} \int_{|y|=1} b_{i j}^{2}(1, y, \xi) f(t-s, x-s y, \xi) \frac{\mathrm{d} S_{y}}{4 \pi s} \mathrm{~d} s \mathrm{~d} \xi .
\end{aligned}
$$

For the first term in the right-hand side, we write

$$
\begin{aligned}
& \left|\int_{0|y|=1}^{\theta_{t}} \int_{i j} b_{i j}^{2}(1, y, \xi)(f(t-s, x-s y, \xi)-f(t-s, x, \xi)) \frac{\mathrm{d} S_{y}}{4 \pi s} \mathrm{~d} s\right| \\
& \quad \leqslant \theta_{t}\left\|b_{i j}^{2}(1, \cdot, \xi)\right\|_{L^{\infty}\left(\mathbf{S}^{2}\right)}\left\|\nabla_{x} f\right\|_{L^{\infty}\left([0, t) \times \mathbf{R}^{6}\right)}
\end{aligned}
$$

$\overline{2}$ p.v. stands for principal value. 
For the second term, we have

$$
\left|\int_{\theta_{t}|y|=1}^{t} \int_{i j}^{2}(1, y, \xi) f(t-s, x-s y, \xi) \frac{\mathrm{d} S_{y}}{4 \pi s} \mathrm{~d} s\right| \leqslant \ln \left(\frac{t}{\theta_{t}}\right)\left\|b_{i j}^{2}(1, \cdot, \xi)\right\|_{L^{\infty}\left(\mathbf{S}^{2}\right)}\|f\|_{L^{\infty}\left([0, t] \times \mathbf{R}^{6}\right)} .
$$

Thus if we choose

$$
\theta_{t}=\inf \left(\frac{1}{\left\|\nabla_{x} f\right\|_{L^{\infty}\left([0, t] \times \mathbf{R}^{6}\right)}}, t\right)
$$

we get

$$
\begin{aligned}
\left|S_{0}\right| \leqslant & C r^{* 3}\|m\|_{L^{\infty}}\left[\left\|c_{i j}\right\|_{L^{\infty}\left(B\left(0, r^{* 3}\right)\right)}\|f\|_{L^{\infty}\left([0, t] \times \mathbf{R}^{6}\right)}+\left\|b_{i j}^{2}\right\|_{L^{\infty}\left(\mathbf{S}^{2} \times \mathbf{R}^{3}\right)}\right. \\
& \left.\times\left(1+\|f\|_{L^{\infty}\left([0, t] \times \mathbf{R}^{6}\right)} \ln \left(1+t\left\|\nabla_{x} f\right\|_{L^{\infty}\left([0, t] \times \mathbf{R}^{6}\right)}\right)\right)\right] .
\end{aligned}
$$

In view of (3.3), this gives

$$
\left|S_{0}\right| \leqslant C\left(f_{I}, \phi_{I}, \phi_{I}^{\prime}, \tau, r^{*}\right)\left(1+\ln \left(1+t\left\|\nabla_{x} f\right\|_{L^{\infty}\left([0, t] \times \mathbf{R}^{6}\right)}\right)\right) .
$$

Estimates for $S_{1}$. This term is very similar to the one arising from the second part of the right-hand side of (3.10). We find

$$
\begin{aligned}
S_{1}= & \int m(\xi)\left(\left(b_{i j}^{1} Y(t, \cdot)\right) \star_{x} f_{I}\right)(x, \xi) \mathrm{d} \xi+\int\left(\left(-\nabla_{\xi}\left(m b_{i j}^{1}\right) Y\right) \star\left(f \mathbf{1}_{t>0} K_{u}\right)\right)(t, x, \xi) \mathrm{d} \xi \\
& +\int\left(\left(m b_{i j}^{1} Y\right) \star\left(f \mathbf{1}_{t>0} T \phi_{u}\right)\right)(t, x, \xi) \mathrm{d} \xi .
\end{aligned}
$$

The only difference with the estimates following (3.10) is the fact that $b_{i j}^{1} \in \mathcal{M}_{-1}$ whereas $a_{i}^{0} \in \mathcal{M}_{0}$. Consequently,

$$
\begin{aligned}
\left|S_{1}\right| \leqslant & \frac{4}{3} \pi r^{* 3}\left(\left\|m t b_{i j}^{1}\right\|_{L^{\infty}}\left\|f_{I}\right\|_{L^{\infty}}+\left\|m t b_{i j}^{1}\right\|_{L_{t, x}^{\infty}\left(W_{\xi}^{1, \infty}\right)} \int_{0}^{t}\left\|f K_{u}(s, \cdot, \cdot)\right\|_{L^{\infty}} \mathrm{d} s\right. \\
& \left.+\left\|m t b_{i j}^{1}\right\|_{L^{\infty}} \int_{0}^{t}\left\|f T \phi_{u}(s, \cdot, \cdot)\right\|_{L^{\infty}} \mathrm{d} s\right) .
\end{aligned}
$$

With (3.3), (3.11) and (3.13), we infer that $S_{1}$ is bounded by a constant:

$$
\left|S_{1}\right| \leqslant C\left(f_{I}, \phi_{I}, \phi_{I}^{\prime}, \tau, r^{*}\right) \text {. }
$$

Estimates for $S_{2}$. This last term requires lengthy computations but the strategy remains the same as above: our goal is to avoid differentiating $f$ by using Eq. (1.12). Let us start with

$$
\begin{aligned}
T^{2}\left(f \mathbf{1}_{t>0}\right)= & T\left(\delta_{t=0} f_{I}\right)+T\left(\mathbf{1}_{t>0}\left(\nabla_{\xi} \cdot\left(f K_{u}\right)+f T \phi_{u}\right)\right) \\
= & \delta_{t=0}^{\prime} f_{I}+\delta_{t=0}\left(v \cdot \nabla_{x} f_{I}+\nabla_{\xi} \cdot\left(f_{I} K_{u}^{I}\right)+f_{I} \phi_{I}^{\prime}+f_{I} v \cdot \nabla_{x} \phi_{I}\right) \\
& +\mathbf{1}_{t>0} T\left(\nabla_{\xi} \cdot\left(f K_{u}\right)\right)+\mathbf{1}_{t>0} T\left(f T \phi_{u}\right) .
\end{aligned}
$$

Working on the last two terms, we find:

$$
\begin{aligned}
T\left(\nabla_{\xi} \cdot\left(f K_{u}\right)\right) & =\nabla_{\xi} \cdot\left(f T K_{u}+\left(\nabla_{\xi} \cdot\left(f K_{u}\right)+f T \phi_{u}\right) K_{u}\right)+\left[T, \nabla_{\xi} \cdot\right]\left(f K_{u}\right) \\
& =\nabla_{\xi} \cdot\left(f T K_{u}+f\left(T \phi_{u}\right) K_{u}\right)+\nabla_{\xi}^{\otimes 2}: f K_{u}^{\otimes 2}-\left(\nabla_{\xi} v\right)^{T}: \nabla_{x}\left(f K_{u}\right) .
\end{aligned}
$$


Note that the last term, which arises from the commutator, will require further computations. Besides,

$$
\begin{aligned}
T\left(f T \phi_{u}\right) & =(T f) T \phi_{u}+f T^{2} \phi_{u} \\
& =\nabla_{\xi} \cdot\left(f K_{u}\right) T \phi_{u}+f\left(T \phi_{u}\right)^{2}+f T^{2} \phi_{u} \\
& =\nabla_{\xi} \cdot\left(f\left(T \phi_{u}\right) K_{u}\right)-\left(f K_{u}\right) \cdot \nabla_{\xi}\left(T \phi_{u}\right)+f\left(T \phi_{u}\right)^{2}+f T^{2} \phi_{u} \\
& =\nabla_{\xi} \cdot\left(f\left(T \phi_{u}\right) K_{u}\right)-\left(\left(f K_{u}\right) \cdot \nabla_{\xi} v\right) \cdot \nabla_{x} \phi_{u}+f\left(T \phi_{u}\right)^{2}+f T^{2} \phi_{u} .
\end{aligned}
$$

This leads to the following decomposition:

$$
\begin{aligned}
T^{2}\left(f \mathbf{1}_{t>0}\right)= & \delta_{t=0}^{\prime} f_{I}+\delta_{t=0}\left(v \cdot \nabla_{x} f_{I}+\nabla_{\xi} \cdot\left(f_{I} K_{u}^{I}\right)+f_{I} \phi_{I}^{\prime}+f_{I} v \cdot \nabla_{x} \phi_{I}\right) \\
& +\mathbf{1}_{t>0} \nabla_{\xi} \cdot\left(f T K_{u}+2 f\left(T \phi_{u}\right) K_{u}\right)+\mathbf{1}_{t>0} \nabla_{\xi}^{\otimes 2}: f K_{u}^{\otimes 2} \\
& -\left(\nabla_{\xi} v\right)^{T}: \nabla_{x}\left(f \mathbf{1}_{t>0} K_{u}\right)-f \mathbf{1}_{t>0}\left(K_{u} \cdot \nabla_{\xi} v\right) \cdot \nabla_{x} \phi_{u}+f \mathbf{1}_{t>0}\left(T^{2} \phi_{u}+\left(T \phi_{u}\right)^{2}\right) .
\end{aligned}
$$

We are now ready to integrate in the $\xi$ variable. The corresponding derivatives are removed by integrating by parts. Thus $S_{2}$ can be written as a sum $S_{20}^{\prime}+S_{20}+S_{21}+S_{22}+S_{23}+S_{24}+S_{25}$ with

$$
\begin{aligned}
& S_{20}^{\prime}=\int m(\xi)\left(b_{i j}^{0} Y\right) \star\left(\delta_{t=0}^{\prime} f_{I}\right) \mathrm{d} \xi \\
& S_{20}=\int m(\xi)\left(b_{i j}^{0} Y\right) \star\left(\delta_{t=0}\left(v \cdot \nabla_{x} f_{I}+\nabla_{\xi} \cdot\left(f_{I} K_{u}^{I}\right)+f_{I} \phi_{I}^{\prime}+f_{I} v \cdot \nabla_{x} \phi_{I}\right)\right) \mathrm{d} \xi \\
& S_{21}=\int\left(-\nabla_{\xi}\left(m b_{i j}^{0}\right) Y\right) \star\left(f \mathbf{1}_{t>0}\left(T K_{u}+2\left(T \phi_{u}\right) K_{u}\right)\right)(t, x, \xi) \mathrm{d} \xi \\
& S_{22}=\int\left(\nabla_{\xi}^{\otimes 2}\left(m b_{i j}^{0} Y\right) \star\left(f \mathbf{1}_{t>0} K_{u}^{\otimes 2}\right)\right)(t, x, \xi) \mathrm{d} \xi \\
& S_{23}=\int m(\xi)\left(\left(\nabla_{\xi} v \cdot \nabla_{x}\left(b_{i j}^{0} Y\right)\right) \star\left(f \mathbf{1}_{t>0} K_{u}\right)\right)(t, x, \xi) \mathrm{d} \xi \\
& S_{24}=\int m(\xi)\left(\left(b_{i j}^{0} Y\right) \star\left(f \mathbf{1}_{t>0}\left(K_{u} \cdot \nabla_{\xi} v\right) \cdot \nabla_{x} \phi_{u}\right)\right)(t, x, \xi) \mathrm{d} \xi \\
& S_{25}=\int m(\xi)\left(\left(b_{i j}^{0} Y\right) \star\left(f \mathbf{1}_{t>0}\left(T^{2} \phi_{u}+\left(T \phi_{u}\right)^{2}\right)\right)(t, x, \xi) \mathrm{d} \xi\right.
\end{aligned}
$$

The first two terms only involve initial data. They are estimated by

$$
\begin{aligned}
\left|S_{20}^{\prime}+S_{20}\right| \leqslant & \frac{4}{3} \pi r^{* 3}\left\|m b_{i j}^{0}\right\|_{L_{x}^{\infty}\left(W_{t, \xi}^{1, \infty}\right)}(1+t)^{2}\left\|f_{I}\right\|_{W^{1, \infty}} \\
& \times\left(1+\left\|K_{u}^{I}\right\|_{L^{\infty}\left(R^{3} \times B\left(0, r^{*}\right)\right)}+\left\|\phi_{I}\right\|_{W^{1, \infty}}+\left\|\phi_{I}^{\prime}\right\|_{L^{\infty}}\right) .
\end{aligned}
$$

The third, fourth, sixth and last terms are estimated in a familiar way:

$$
\begin{aligned}
& \left|S_{21}\right| \leqslant \frac{4}{3} \pi r^{* 3}\left\|m b_{i j}^{0}\right\|_{L_{t, x}^{\infty}\left(W_{\xi}^{1, \infty}\right)} \int_{0}^{t}(t-s)\left\|f\left(T K_{u}+2\left(T \phi_{u}\right) K_{u}\right)(s, \cdot, \cdot)\right\|_{L^{\infty}} \mathrm{d} s, \\
& \left|S_{22}\right| \leqslant \frac{4}{3} \pi r^{* 3}\left\|m b_{i j}^{0}\right\|_{L_{t, x}^{\infty}\left(W_{\xi}^{2, \infty}\right)} \int_{0}^{t}(t-s)\left\|f K_{u}^{\otimes 2}(s, \cdot, \cdot)\right\|_{L^{\infty}} \mathrm{d} s, \\
& \left|S_{24}\right| \leqslant \frac{4}{3} \pi r^{* 3}\left\|m b_{i j}^{0}\right\|_{L^{\infty}} \int_{0}^{t}(t-s)\left\|f\left(K_{u} \cdot \nabla_{\xi} v\right) \cdot \nabla_{x} \phi_{u}(s, \cdot, \cdot)\right\|_{L^{\infty}} \mathrm{d} s,
\end{aligned}
$$




$$
\left|S_{25}\right| \leqslant \frac{4}{3} \pi r^{* 3}\left\|m b_{i j}^{0}\right\|_{L^{\infty}} \int_{0}^{t}(t-s)\left\|f\left(T^{2} \phi_{u}+\left(T \phi_{u}\right)^{2}\right)(s, \cdot, \cdot)\right\|_{L^{\infty}} \mathrm{d} s .
$$

Expression (1.10) shows that

$$
\left\|T K_{u}(s, \cdot, \cdot)\right\|_{\left.L^{\infty}\left(\mathbf{R}^{3} \times B\left(0, r^{*}\right)\right)\right)} \leqslant C\left(r^{*}\right) J(s) .
$$

Using estimates (3.3) and (3.13), it comes then

$$
\left|S_{21}+S_{22}+S_{24}+S_{25}\right| \leqslant C\left(f_{I}, \phi_{I}, \phi_{I}^{\prime}, \tau, r^{*}\right)\left(1+\int_{0}^{t} J(s) \mathrm{d} s\right) .
$$

As said above, the remaining term $S_{23}$ requires an additional step. We brought the derivatives to the left side of the convolution in order to use Lemma 2.1 one more time. We have

$$
\partial_{k}\left(b_{i j}^{0} Y\right)=T\left(b_{i j}^{0} a_{k}^{0} Y\right)+\left(b_{i j}^{0} a_{k}^{1}-a_{k}^{0} T\left(b_{i j}^{0}\right)+\partial_{k} b_{i j}^{0}\right) Y,
$$

which yields

$$
\nabla_{\xi} v \cdot \nabla_{x}\left(b_{i j}^{0} Y\right)=T\left(c_{i j}^{0} Y\right)+c_{i j}^{1} Y,
$$

where we set

$$
\begin{aligned}
& c_{i j}^{0}=b_{i j}^{0} \nabla_{\xi} v \cdot a^{0}, \\
& c_{i j}^{1}=b_{i j}^{0} \nabla_{\xi} v \cdot a^{1}-\left(\nabla_{\xi} v \cdot a^{0}\right) T b_{i j}^{0}+\nabla_{\xi} v \cdot \nabla_{x} b_{i j}^{0} .
\end{aligned}
$$

Therefore $S_{23}$ can be written as

$$
S_{23}=\int m(\xi)\left(\left(c_{i j}^{0} Y\right) \star T\left(f \mathbf{1}_{t>0} K_{u}\right)\right)(t, x, \xi) \mathrm{d} \xi+\int m(\xi)\left(\left(c_{i j}^{1} Y\right) \star\left(f \mathbf{1}_{t>0} K_{u}\right)\right)(t, x, \xi) \mathrm{d} \xi .
$$

Using another time the transport equation,

$$
T\left(f \mathbf{1}_{t>0} K_{u}\right)=f_{I} K_{u}^{I} \delta_{t=0}+\mathbf{1}_{t>0} f T K_{u}+\mathbf{1}_{t>0} \nabla_{\xi} \cdot\left(f K^{\otimes 2}\right)-\mathbf{1}_{t>0} f\left(K_{u} \cdot \nabla_{\xi}\right) K_{u}+f\left(T \phi_{u}\right) K_{u},
$$

it is now routine work to see that

$$
\left|S_{23}\right| \leqslant C\left(f_{I}, \phi_{I}, \phi_{I}^{\prime}, \tau, r^{*}\right)\left(1+\int_{0}^{t} J(s) \mathrm{d} s\right) .
$$

Using (3.13) and gathering the inequalities above, we infer that

$$
\left|S_{2}\right| \leqslant C\left(f_{I}, \phi_{I}, \phi_{I}^{\prime}, \tau, r^{*}\right)\left(1+\int_{0}^{t} J(s) \mathrm{d} s\right) .
$$

Collecting estimates (3.14), (3.16), (3.17) and (3.18),

$$
J(t) \leqslant C\left(f_{I}, \phi_{I}, \phi_{I}^{\prime}, \tau, r^{*}\right)\left(1+\ln \left(1+t\left\|\nabla_{x} f\right\|_{L^{\infty}\left([0, t] \times \mathbf{R}^{6}\right)}\right)+\int_{0}^{t} J(s) \mathrm{d} s\right)
$$

for any $0<t<\tau$. Applying Gronwall's lemma, we get for $0<t<\tau$,

$$
J(t) \leqslant C\left(f_{I}, \phi_{I}, \phi_{I}^{\prime}, \tau, r^{*}\right) \ln \left(1+t\left\|\nabla_{x} f\right\|_{L^{\infty}\left([0, t] \times \mathbf{R}^{6}\right)}\right) .
$$

Note that the behaviour of this bound is governed by the contribution from the most singular term, namely $S_{0}$. 


\subsection{Proof of Theorem 1.1}

With (3.9) and (3.13), (3.19) yields

$$
\left\|\phi_{u}\right\|_{W^{2, \infty}\left([0, t] \times \mathbf{R}^{3}\right)} \leqslant C\left(f_{I}, \phi_{I}, \phi_{I}^{\prime}, \tau, r^{*}\right)\left(1+\ln \left(1+\|f\|_{W^{1, \infty}\left([0, t] \times \mathbf{R}^{6}\right)}\right)\right) .
$$

Using this in (3.5) gives

$$
\|f(t, \cdot, \cdot)\|_{W^{1, \infty}} \leqslant C\left(f_{I}, \phi_{I}, \phi_{I}^{\prime}, \tau, r^{*}\right)\left(1+\int_{0}^{t}\|f(s, \cdot, \cdot)\|_{W^{1, \infty}}\left(1+\ln \left(1+\|f\|_{W^{1, \infty}\left([0, s] \times \mathbf{R}^{6}\right)}\right)\right) \mathrm{d} s\right) .
$$

The growth rate in this estimate is decisive and allows the use of a logarithmic Gronwall's lemma, showing that

$$
\|f\|_{W^{1, \infty}\left([0, \tau) \times \mathbf{R}^{6}\right)} \leqslant C\left(f_{I}, \phi_{I}, \phi_{I}^{\prime}, \tau, r^{*}\right) .
$$

We eventually infer from (3.20) the expected estimate

$$
\left\|\phi_{u}\right\|_{W^{2, \infty}\left([0, \tau) \times \mathbf{R}^{6}\right)} \leqslant C\left(f_{I}, \phi_{I}, \phi_{I}^{\prime}, \tau, r^{*}\right) .
$$

This ends the proof of Theorem 1.1.

\section{References}

[1] H. Andréasson, S. Calogero, G. Rein, Global classical solutions to the spherically symmetric Nordström-Vlasov system, Math. Proc. Cambridge Philos. Soc., in press.

[2] F. Bouchut, F. Golse, C. Pallard, Nonresonant smoothing for coupled wave + transport equations and the Vlasov-Maxwell system, Rev. Mat. Iberoamericana, in press.

[3] F. Bouchut, F. Golse, C. Pallard, Classical solutions and the Glassey-Strauss theorem for the 3D Vlasov-Maxwell System, Arch. Rational Mech. Anal. 170 (2003) 1-15.

[4] S. Calogero, Spherically symmetric steady states of galactic dynamics in scalar gravity, Class. Quantum Grav. 20 (2003) $1729-1741$.

[5] S. Calogero, H. Lee, The non-relativistic limit of the Nordström-Vlasov system, Comm. Math. Sci. 2 (2004) 19-34.

[6] S. Calogero, G. Rein, On classical solutions of the Nordström-Vlasov system, Comm. Partial Differential Equations 28 (2003) $1863-1885$.

[7] S. Calogero, G. Rein, Global weak solutions to the Nordström-Vlasov system, J. Differential Equations, in press.

[8] S. Friedrich, Global small solutions of the Vlasov-Nordström system, math-ph/0407023.

[9] I. Gelfand, G. Shilov, Generalized Functions. Vol. I, Academic Press, New York, 1964.

[10] R. Glassey, W. Strauss, Singularity formation in a collisionless plasma could occur only at high velocities, Arch. Rational Mech. Anal. 92 (1986) 59-90.

[11] S. Hawking, G. Ellis, The Large Scale Structure of Space-Time, Cambridge Monographs Math. Phys., Cambridge University Press, 1973.

[12] L. Hörmander, The Analysis of Linear Partial Differential Operators. I. Distribution Theory and Fourier Analysis, Springer-Verlag, Berlin, 1983.

[13] S. Klainerman, G. Staffilani, A new approach to study the Vlasov-Maxwell system, Commun. Pure Appl. Anal. 1 (2002) $103-125$.

[14] H. Lee, Global existence of solutions to the Nordström-Vlasov system in two space dimensions, math-ph/0312014. 